\title{
Erhöhtes perioperatives Schlaganfallrisiko bei Patienten mit Migräne
}

Fragestellung: Besteht bei Patienten mit Migräne ein erhöhtes Risiko, perioperativ einen ischämischen Schlaganfall zu erleiden?

Hintergrund: Bis zu $20 \%$ aller Menschen in Europa und Nordamerika leiden unter einer Migräne. Seit langem ist bereits bekannt, dass die Migräne, insbesondere die Migräne mit Aura, bei Frauen mit einem erhöhten Schlaganfallrisiko einhergeht. Perioperative Schlaganfälle (bis zu 30 Tage nach einer operativen Intervention) treten bei $0,1 \%$ aller allgemeinchirurgischen Eingriffe auf und in 1-7\% der Patienten nach Herz- oder Gefäßoperationen. Vor dem Hintergrund, dass in den USA 50 Millionen operative Eingriffe im Krankenhaus erfolgen, ist dies ein nicht unerhebliches Risiko. Bisher war nicht untersucht worden, ob das Risiko perioperativer Schlaganfälle bei Patienten mit Migräne erhöht ist.

Patienten und Methodik: Die Studie stützt sich auf das Krankenhausregister des Massachusetts General Hospital mit einer Datenerhebung zwischen Januar 2007 und August 2014. Erfasst wurden 124.558 chirurgische

Timm FP, Houle TT, Grabitz SD et al. Migraine and risk of perioperative ischemic stroke and hospital readmission: hospital based registry study. BMJ 2017; 356: i6635 Eingriffe. Die Patienten waren im Mittel 53 Jahre alt und $55 \%$ waren Frauen.

Der primäre Endpunkt der Studie waren perioperative ischämische Schlaganfälle, die bis zu 30 Tage nach einem chirurgischen Eingriff auftreten. Verglichen wurden Patienten mit und ohne Migräne. Der sekundäre Outcome-Parameter war eine erneute Krankenhausaufnahme innerhalb von 30 Tagen nach einer Operation.

Ergebnisse: Von den 124.558 Patienten, die operiert wurden, hatten $10.179(8,2 \%)$ eine Migräne. Davon wiesen 1.278 Patienten eine Migräne mit Aura auf. Innerhalb von 30 Tagen nach dem operativen Eingriff betrug das Risiko für einen ischämischen Insult 0,6\%. Patienten, die unter einer Migräne litten, hatten einen um den Faktor 1,75 erhöhtes Risiko einen perioperativen ischämischen Schlaganfall zu erleiden verglichen mit $\mathrm{Pa}$ tienten ohne Migräne. Das Risiko war nochmals erhöht bei Patienten die unter einer Migräne mit Aura litten (Odds Ratio 2,61). Das absolute Risiko, einen ischämischen Insult zu erleiden, betrug 2,4 pro 1.000 chirurgische Eingriffe. Das absolute Risiko stieg auf 4,3 pro 1.000 bei Migräne und auf 6,3 bei Migräne mit Aura.

Auch der sekundäre Outcome wurde durch die Migräne beeinflusst: Migränepatienten wurden häufiger innerhalb von 30 Tagen nach der Operation wieder im Krankenhaus aufgenommen.

Schlussfolgerungen: Patienten, die unter einer Migräne leiden und sich einem chirurgischen Eingriff unterziehen müssen, haben ein leicht erhöhtes Risiko, innerhalb von 30 Tagen nach dem operativen Eingriff einen ischämischen Schlaganfall zu erleiden.

\section{- Kommentar von Hans-Christoph Diener, Essen}

\section{Liegt eine veränderte Endothelfunktion zugrunde?}

Diese Studie zeigt, dass bei Menschen mit Migräne nicht nur das Risiko vaskulärer Ereignisse wie ischämischer Insult oder zerebrale Blutungen und kardiovaskuläre Ereignisse erhöht ist, sondern offenbar auch das perioperative Schlaganfallrisiko. Grundlage dieser Beobachtung könnte sein, das Patienten mit Migräne eine veränderte Endothelfunktion haben oder auf den operativen und perioperativen Stress anders reagieren, als Menschen ohne eine Migräne.

In der vorliegenden Studie war das Risiko bei Menschen die eine Migräne mit Aura haben, signifikant höher als bei Menschen mit Migräne ohne Aura. Dies entspricht der Beobachtung, dass auch generell das Schlaganfallrisiko bei Migräne mit Aura erhöht ist. Ungeklärt ist allerdings die Frage, welche praktischen Konsequenzen sich aus dieser Studie ergeben. Solange der pathophysiologische Mechanismus des erhöhten Schlaganfallrisikos nicht geklärt ist, können praktisch auch keine präventiven Maßnahmen zur Reduktion des Schlagan- fallrisikos ergriffen werden. Eine mögliche Option wäre aber, Substanzen während der Operation zu vermeiden, die negative Effekte auf die Endothelfunktion haben.

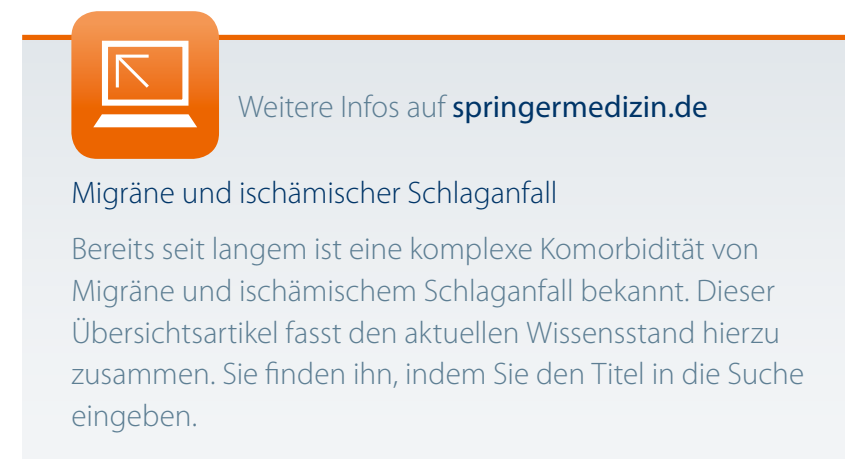

